\title{
Quaderni
}

QUADERNI Communication, technologies, pouvoir

97 | Automne 2018

Néo-libéralisme(s). Réseaux et formes des

mobilisations en France

\section{Néo-libéralisme(s).Réseaux et formes des mobilisations en France}

Avant-propos

\section{Kevin Brookes}

\section{(2) OpenEdition \\ Journals}

\section{Édition électronique}

URL : http://journals.openedition.org/quaderni/1225

DOI : 10.4000/quaderni.1225

ISSN : 2105-2956

\section{Éditeur}

Les éditions de la Maison des sciences de l'Homme

Édition imprimée

Date de publication : 5 octobre 2018

Pagination : 05-13

\section{Référence électronique}

Kevin Brookes, « Néo-libéralisme(s). Réseaux et formes des mobilisations en France », Quaderni [En ligne], 97 | Automne 2018, mis en ligne le 05 octobre 2018, consulté le 04 janvier 2021. URL : http:// journals.openedition.org/quaderni/1225; DOI : https://doi.org/10.4000/quaderni.1225 


\section{$D$ ossier}

\section{Avant-propos Néo-libéralisme(s). Réseaux et formes des mobilisations} en France

\section{Kevin Brookes}

Université Grenoble Alpes Science Po Grenoble / PACTE
La diffusion du néo-libéralisme est l'un des faits politiques marquants de la fin des années 1970, en raison des transformations qu'il a engendrées et des résistances qu'il a suscitées. Celle-ci a accompagné une "grande transformation», à la fois intellectuelle et politique, dans le rapport des démocraties au marché et dans les conceptions de l'action publique des principaux dirigeants $^{1}$. Il constitue le versant intellectuel de la crise du keynésianisme des années 1970 et marque une rupture paradigmatique dans le domaine des politiques économiques dans les pays occidentaux. Ses avocats ont contribué à hisser le thème de la crise de l'État-providence au premier plan des discussions politiques et universitaires ${ }^{2}$. La crise économique de 2008 n'a fait qu'alimenter les débats sur ses avatars et la question de sa résilience ${ }^{3}$.

L'étude du néo-libéralisme est aujourd'hui à la mode et le foisonnement de la production académique mobilisant la notion n'a pas contribué à l'éclaircir bien au contraire : le mot est devenu un concept «attrape-tout» et «flou » faisant l'économie d'une définition claire 4 . Il est aussi devenu un principe explicatif de phénomènes très différents (la dégradation de l'université, l'épuisement de l'État-providence, l'hypersollicitation des individus, les fermetures d'usine, etc.). La littérature souffre d'un deuxième travers qui est l'imprécision dans l'emploi de ce terme, qui n'a pourtant jamais autant été mobilisé, mais qui n'est que trop peu souvent défini. Taylor Boas et Jordan Gans-Morse ont dressé ce double constat sur le caractère flou et chargé idéologiquement du mot à partir d'une étude bibliométrique portant sur un corpus de publications académiques internationales au début des années $2000^{5}$. 
Ils remarquent d'abord que le terme est de plus en plus employé : il était mobilisé dans 1000 articles académiques par an entre 2002 et 2005 , alors qu'il n'était quasiment pas employé avant les années 1990. Il n'était défini que dans $30 \%$ des cas et n'avait une connotation positive que dans $3 \%$ d'entre eux ( $45 \%$ neutre, $45 \%$ négative, $8 \%$ avec les deux connotations). Cette instabilité définitionnelle autour du concept de néolibéralisme nous invite à le définir préalablement avant de présenter l'originalité de la démarche de ce numéro spécial de Quaderni pour la compréhension des formes de mobilisation autour du néo-libéralisme en France.

\section{Qu'est-ce que le néo-libéralisme?}

Le néo-libéralisme est issu d'un processus de théorisation. L'enjeu est de saisir le mouvement qui va d'une théorisation préalable à une appropriation par des acteurs sociaux. Nous proposons ici de définir le néo-libéralisme avant tout par les idées des penseurs qui l'ont formulé, et en prenant en considération la diversité de ses écoles. Il faut prendre en compte également les réseaux et les formes de la diffusion de cette idéologie. Il est nécessaire de lire les auteurs ayant théorisé cette doctrine afin de saisir les deux bouts de la chaîne lorsque l'on analyse les idéologies dans une perspective de sociologie politique. Pour étudier la production intellectuelle, il faut d'abord se familiariser avec l'analyse de ses théoriciens, leur hétérogénéité, leurs positions par rapport à d'autres, les logiques intellectuelles de la doctrine qu'ils élaborent. Il s'agit d'une étape indispensable afin de mieux appréhender ensuite les usages de la doctrine dans l'espace public en repérant les éléments de langage, les notions, les références mobilisées et analyser ainsi les conditions sociales de nature à expliquer les écarts existants entre le producteur d'un texte et ses lecteurs. Les travaux en sociologie de la lecture ont en effet montré que le lecteur n'était pas un simple récepteur, mais était également acteur de sa réappropriation subjective en opérant une lecture sélective au sein de «communautés interprétatives $^{6}$. \»

Le néo-libéralisme doit être, à notre sens, caractérisé comme une idéologie, c'est-à-dire un ensemble d'idées à la fois descriptives et prescriptives qui orientent le comportement politique des acteurs en leur donnant un sens ${ }^{7}$. Pour reprendre la distinction de John Campbell, les idées peuvent être cognitives (analysant des causes et décrivant le réel) ou normatives (énoncer ce qui doit être) ${ }^{8}$. Les idées néo-libérales ont cette double nature. Ainsi, le néo-libéralisme doit être analysé dans sa dimension duale : à la fois comme un programme de recherche s'appuyant sur des présupposés épistémologiques fondant une analyse positive de la société, et comme un ensemble de théories normatives impliquant la mise en œuvre de certaines politiques publiques en découlant 9 .

Les cours de Michel Foucault sur la naissance de la biopolitique sont extrêmement précieux pour nous aider à définir le néo-libéralisme contemporain en distinguant ses deux principales tendances et distinguer le néo-libéralisme du libéralisme classique. Foucault considérait, dans la lignée de ses travaux sur le pouvoir, le libéralisme comme un ensembles de savoirs, méthodes et instruments de réflexions qui théorisait la limitation interne de la raison gouvernementale fondée à la fois sur la science économique et 
le droit ${ }^{10}$. Cette «frugalité du gouvernement» était pensée par les économistes (l'utilité) qui se fondaient sur un ensemble de théories faisant du marché un lieu de véridiction et par les juristes (droit naturel) qui basaient leur argumentation sur la défense des droits de l'homme.

Foucault remarque, au sein du libéralisme qui se renouvelle à partir des années 1930, l'existence de deux «programmations libérales », de deux néolibéralismes : un ancrage allemand et un autre américain ${ }^{11}$. À propos du premier, il montre à quel point ses théoriciens ont légitimé les interventions de l'État pour assurer les conditions d'une concurrence pure et parfaite en se posant en rupture avec le « laisser-faire ». Il montre également que le néo-libéralisme américain est autrement plus radical et comporte des différences de fond massives en ce qu'il constitue une « grande alternative économico-politique, sinon de masse, du moins très largement répandue à l'intérieur de la société américaine ${ }^{12}$. » C'est pourquoi il explique que ces deux phénomènes ne sont pas superposables et ne peuvent s'étudier ensemble.

Après avoir pris soin de déhomogénéiser le néo-libéralisme, nous pouvons en proposer une définition englobante et caractériser son préfixe. Il s'agit d'une idéologie critiquant l'intervention de l'État dans l'économie et consacrant le marché libre comme l'institution la plus efficace et la plus juste pour organiser la coordination des individus en société. Cette définition ne semble pas différer de la doctrine du laisser-faire du $\mathrm{XIX}^{\mathrm{e}}$ siècle incarnée par exemple par l'école d'économie politique française ou par l'école de Manchester. En quoi donc le néo-libéralisme serait-il «néo » et différerait-il du libéralisme clas- sique, parfois qualifié, à escient, par ses promoteurs et ses détracteurs, de « paléo-libéralisme»? Dans son sens contemporain, le préfixe « néo » renvoie, selon nous, davantage à une dimension de renouveau, plutôt qu'à la dimension réformatrice de la doctrine qu'elle avait lorsque le mot est apparu dans les années 1930. La «nouveauté» implicite dans le préfixe « néo » de cette doctrine renvoie à trois éléments : le contexte historique défavorable dans lequel ces théories s'élaborent, la nouvelle scientificité dont se parent certaines de ses écoles, et une radicalisation qui se traduit par la volonté de généraliser les mécanismes de marché dans tous les domaines (social, politique, culturel).

Enfin, si le néo-libéralisme est difficile à saisir et à définir, c'est qu'il s'exprime dans différents domaines, conférant trois facettes à cette idéo$\operatorname{logie^{13}}$. Il présente d'abord une facette intellectuelle qui prend la forme d'un projet idéologique transnational théorisé par des intellectuels. Elle renvoie aux projets intellectuels des théoriciens de cette idéologie et leurs réseaux pour diffuser leurs savoirs.

Le néo-libéralisme présente également une facette politique au sens de Max Weber en renvoyant aux «efforts que l'on fait en vue de participer au pouvoir ou d'influencer la répartition du pouvoir, soit entre les États, soit entre les divers groupes à l'intérieur d'un même État ${ }^{14}$.» Elle s'incarne dans des organisations ou entrepreneurs politiques dont l'objectif est de mobiliser les électeurs et remporter les élections en s'appuyant sur une rhétorique néo-libérale pour contrer ses adversaires ou proposer un programme inspiré par cette idéologie. Les idées néo-libérales ont été portées par des 
entrepreneurs politiques qui s'en sont servis comme des armes permettant de remettre en cause les arrangements institutionnels existants ${ }^{15}$.

Enfin, le néo-libéralisme présente une facette administrative qui s'incarne dans des politiques publiques spécifiques. Il s'agit des recettes proposées en matière économique et social pour transformer les actions de l'État afin de se conformer à l'idéologie néo-libérale : diminution des budgets, ciblage des aides sociales (vouchers), lutte contre l'inflation, suppression du salaire minimum....

\section{Une approche compréhensive et incarnée du néo-libéralisme}

Le regain d'intérêt des chercheurs pour l'objet «néo-libéralisme» a ouvert la voie à de nombreuses d'études permettant de mieux comprendre certains de ses aspects. Sa genèse intellectuelle au début du $\mathrm{XX}^{\mathrm{e}}$ siècle, et son institutionnalisation dans des réseaux intellectuels au cours de la seconde moitié du $\mathrm{XX}^{\mathrm{e}}$ sont désormais bien connues ${ }^{16}$. D'un autre côté, la politique comparée a permis de comprendre ses réappropriations différenciées en fonction des contextes politiques et institutionnels nationaux ${ }^{17}$. L'histoire intellectuelle du néolibéralisme a connu également des développements féconds pour le saisir dans toute la diversité de ses écoles ${ }^{18}$.

Cependant, une partie de la littérature est dominée par des recherches se focalisant sur les manifestations supposées du néolibéralisme, qui serait devenu un discours hégémonique ou une «nouvelle raison du monde» gouvernant les actions individuelles et dont la logique s'appliquerait partout ${ }^{19}$.

Face à cette approche globalisante du néo-libéralisme, l'objectif de ce numéro est de proposer une sociologie incarnée du militantisme néolibéral avec des contributions centrées sur les «Pèlerins ${ }^{20} »$, c'est-à-dire sur les acteurs portant ces idées, ainsi que sur leurs actions pour les mettre en application dans le domaine des politiques publiques. Cette démarche compréhensive et analytique permet de retracer les trajectoires des idées et des acteurs qui les portent en restituant leurs intentions et les formes de mobilisation auxquelles ils ont recours pour en faire la promotion. Elle attache également une importance au discours de légitimation de ces acteurs vis-à-vis de leurs formes de mobilisations et la justification de leurs idées. Elle permet, tout en tenant compte des contextes institutionnels et historiques, de prendre les idées défendues par les néo-libéraux «au sérieux», ce qui permet une meilleure appréhension de ce qu'est le néo-libéralisme en pratiques en saisissant sa circulation des sphères militantes aux sphères de pouvoir dans le contexte français ${ }^{21}$.

Les études comparatives existantes sur la diffusion du néo-libéralisme montrent qu'il existe une résistance à l'égard de cette idéologie ${ }^{22}$. On peine à trouver en France des équivalents de figures et mouvements s'appuyant sur une rhétorique faisant l'apologie du marché libre tel qu'aux États-Unis, au Royaume-Uni, en Allemagne ou dans certains pays d'Amérique Latine. Cette mise à l'agenda d'un discours néolibéral et de recettes de politiques publiques visant à laisser libre cours aux mécanismes de marché 
s'est faite en lien avec un réseau international de think tanks, d'intellectuels et d'experts partageant le même objectif de remise en cause du modèle économique et social keynésien d'après-guerre.

Or, peu de travaux et d'évidences empiriques existent dans le cas français pour attester de ces liens avec ce réseau transnational et témoigner d'une influence de membres de ces sociétés internationales sur la politique française ${ }^{23}$.

Ce numéro de Quaderni contribue ainsi à combler un triple manque. Le premier concerne la littérature sur le néo-libéralisme qui tend à privilégier une approche intellectualiste en se concentrant sur les «grands auteurs» (Friedrich Hayek, Milton Friedman, Wilhelm Röpke...) au détriment d'une sociologie de ses «médiateurs». Le rôle de ces derniers est pourtant central car ce sont eux qui transforment la pensée économique en arme politique et retraduisent des théories produites dans l'univers académique des sciences sociales en action politique visant à transformer le réel ${ }^{24}$. Le succès des idées des philosophes vient en effet de la capacité de médiateurs de résumer leurs idées sous la forme d'associations d'idées simples et compréhensibles dans d'autres champs $^{25}$.

Le deuxième manque porte sur la sociologie du militantisme qui s'est concentrée ces dernières années quasiment exclusivement sur des organisations «de gauche» (communistes, socialistes, altermondialistes, féministes...) au détriment d'études sur des mobilisations autour de causes morales ou idéologiques associées au libéralisme ou au conservatisme ${ }^{26}$.

Enfin, peu de travaux sont, à ce jour, consacrés aux militants et aux organisations qui font la promotion du néo-libéralisme dans le contexte français. Ce numéro vise à combler, en partie, ce manque.

Qui sont les médiateurs du néo-libéralisme en France? Quels sont leurs répertoires d'actions, les formes de mobilisations qu'ils privilégient? Quels sont leurs liens avec les acteurs transnationaux de diffusion du néo-libéralisme et comment inscrivent-ils leur idéologie dans le contexte français?

Pour répondre à ces questions, ont été privilégiées les contributions décloisonnant les approches disciplinaires et articulant la sociologie des acteurs et discours néo-libéraux avec le cadre institutionnel et historique dans lequel ils évoluent.

$* * *$

Le premier article du numéro analyse les résistances importantes au néo-libéralisme en montrant la persistance d'un discours anticapitaliste structuré en France. L'historien Émile Chabal part du constat que les Français sont majoritairement hostiles au néo-libéralisme et que les libéraux, de manière générale, ont souvent eu plus d'ennemis que d'alliés dans l'histoire. À partir de l'exégèse de quatre textes, il présente le renouveau du discours anticapitaliste depuis les reconfigurations idéologiques qui ont eu lieu au cours des années 1970. Il constate que l'anticapitalisme, malgré l'effondrement du marxisme, est parvenu à se maintenir en renouvelant ses critiques. Il l'a fait en s'adaptant au contexte socio-politique, et notamment en réexaminant le concept de pou- 
voir dans un monde de plus en plus fragmenté, pluraliste, marqué par un affaiblissement du clivage gauche-droite. Certains de ses concepts, comme la notion de « classe », de « révolution" ont laissé place à de nouveaux concepts autour desquels se mobilisent les forces s'opposant au néo-libéralisme. Émile Chabal montre, à travers ce tour d'horizon, la richesse et la diversité des écoles de pensée qui s'opposent à la facette intellectuelle du néo-libéralisme : écologie politique (André Gorz), républicanisme (Régis Debray), nouvelle sociologie critique (Luc Boltanski et Ève Chiapello), néo-foucaldisme (Pierre Dardot et Christian Laval)... Selon lui cette vigueur intellectuelle n'est pas étrangère aux résistances politiques au néo-libéralisme qui ont pu s'exprimer à partir des années 1990.

La deuxième contribution revient sur un type d'organisation spécifique de promotion du néolibéralisme : le think tank. Notre article mobilise des sources primaires pour retracer les canaux de diffusion de la pensée néo-libérale à travers ces organisations assurant la médiation entre la facette intellectuelle internationale du néolibéralisme et sa facette politique en France dans les années 1980. Il s'appuie sur l'étude de cas de trois organisations partageant le même objectif et tissant des liens avec des think tanks américains et anglais : l'Association pour la liberté économique et sociale (1966), l'Institut Économique de Paris (1982) et Printemps 86 (1984). L'article montre que des réseaux transnationaux comme la Société du Mont Pèlerin et Atlas Economic Network ont joué un rôle essentiel pour favoriser l'émergence de think tanks situés à la croisée entre le savoir et le pouvoir en France. Il met en évidence le cas concret d'un transfert de savoir-faire et de financement d'un think tank britannique vers un think tank français. Les contexte national et international ont favorisé une certaine influence sur les organisations partisanes de droite (alors situées dans l'opposition) en contribuant à transformer leur discours et leur programme économique au cours des années 1980 en s'inspirant de plusieurs exemples à l'étranger. Même si leur rôle doit être relativisé, en raison de leur courte existence et leur faible médiatisation, elles ont fourni des conditions favorables pour l'arrivée d'une nouvelle génération de think tanks au début des années 2000 .

La contribution du sociologue Benjamin Tainturier s'inscrit dans la suite logique de la précédente, en s'interrogeant sur les effets qu'a eu l'essor d'Internet sur les mobilisations en faveur du néo-libéralisme dans sa version utopique (le libertarianisme). Il montre les affinités électives de la technologie d'Internet qui repose sur une organisation décentralisée et réticulaire avec la pensée néo-libérale. Malgré cela, à partir de son analyse quantitative de la structuration des sites internet libertariens en France, il constate la prédominance des organisation néo-libérales ancrées depuis plusieurs années. Le réseau militant libertarien français reste selon lui encore très centralisé. Son travail de terrain auprès de jeunes militants lui permet de constater l'existence d'un écart clivage intergénérationnel important et l'apparition de nouvelles pratiques militantes.

L'entretien avec Gaspard Koenig, directeur du think tank GenerationLibre offre un exemple concret d'une trajectoire militante néo-libérale. Son itinéraire intellectuel révèle une certaine originalité par son adhésion aux principes philo- 
sophiques du libéralisme davantage qu'à ceux de l'économie de marché à strictement parler. Après avoir expérimenté d'autres formes de mobilisation en faveur de ses idées (notamment en se présentant à une élection), le philosophe a considéré que la manière la plus efficace de les défendre était de fonder son propre think tank. L'entretien témoigne, là aussi, de l'existence d'un modèle anglais de think tanks : GenerationLibre s'inspire directement de l'Institute of Economic Affairs et du Center for Political Studies. C'est notamment le cas en ce qui concerne sa stratégie visant à influencer les décideurs publics et les élites médiatiques. Les actions de GénérationLibre démontrent une volonté de rendre l'idéologie néo-libérale respectable auprès des élites administratives en mobilisant le recours à l'expertise technique pour diffuser des idées comme le revenu universel.

Le dernier article de ce numéro spécial analyse la facette administrative du néo-libéralisme à travers l'exemple d'un instrument de politiques publiques : le chèque culture. Le politiste Arnaud Lacheret fait sa généalogie intellectuelle en montrant que, loin de la neutralité politique qu'on lui attribue parfois, cette politique publique individualisant une aide sociale puise ses sources dans la pensée de l'économiste néo-libéral Milton Friedman. Il analyse ensuite le discours des décideurs publics les légitimant à partir d'un corpus d'entretiens et de documents de communication. Il montre qu'à l'origine, dans les premières collectivités territoriales ayant mis en place ce chèque, cette filiation était non seulement assumée mais revendiquée (comme la région RhôneAlpes). L'outil a survécu aux défaites électorales de ses créateurs, mais le discours a profondément évolué en prenant une forme utilitariste permettant de neutraliser le caractère néo-libéral de cette politique publique. 
$\mathrm{N} \cdot \mathrm{O} \cdot \mathrm{T} \cdot \mathrm{E} \cdot \mathrm{S}$

1. Mark Blyth, Great Transformations: Economic Ideas and Institutional Change in the Twentieth Century, Cambridge, Cambridge University Press, 2002.

2. François-Xavier Merrien, L'État-providence, $3^{\mathrm{e}}$ édition entièrement refondue, Paris, Presses Universitaires de France, 2007.

3. Colin Crouch, The strange non-death of neoliberalism, Cambridge, Polity Press, 2011 ; Vivien Ann Schmidt et Mark Thatcher (dir.), Resilient Liberalism in Europe's Political Economy, Cambridge, RoyaumeUni, États-Unis, Cambridge University Press, 2013.

4. Gwendal Châton et Sébastien Caré, « Néolibéralisme (s) et démocratie (s) », Revue de philosophie économique, vol. 17, $\mathrm{n}^{\circ}$ 1, 2016, p. 3.

5. Taylor C. Boas et Jordan Gans-Morse, "Neoliberalism: From New Liberal Philosophy to Anti-Liberal Slogan", Studies in Comparative International Development, vol. 44, n 2, 2009, p. 138.

6. Stanley Eugene Auteur Fish, Is There a Text in this Class?: The Authority of Interpretive Communities, Cambridge (Mass.), Harvard University Press, 1980. 7. Michael Freeden, Ideology: a very short introduction, Oxford University Press, 2003.

8. John L. Campbell, "Institutional analysis and the role of ideas in political economy", Theory and society, vol. 27, n 3, 1998, p. 377-409.

9. Maurice Lagueux, «Le néo-libéralisme comme programme de recherche et comme idéologie », Cahiers d'économie politique, no 16-17, 1989, p. 129-152.

10. Michel Foucault, Naissance de la biopolitique: cours au Collège de France (1978-1979), Paris, Gallimard, 2004, p. 12.

11. Ibid., p. 80.

12. Ibid., p. 197.

13. Nous empruntons cette distinction à Stéphanie Lee
Mudge, "What is neo-liberalism?", Socio-Economic Review, vol. 6, n 4, 2008, p. 703-731.

14. Max Weber, Le savant et le politique, UQAM - Les Classiques des Sciences Sociales (édition numérique), 1919, p. 87.

15. Mark Blyth, "The transformation of the Swedish model: Economic ideas, distributional conflict, and institutional change", World Politics, vol. 54, $\mathrm{n}^{\circ} 1$, 2001, p. 1-26.

16. Angus Burgin, The great persuasion: reinventing free markets since the Depression, Cambridgen, Harvard University Press, 2012 ; Philipp Mirowski et Dieter Plehwe (dir.), The Road from Mont Pèlerin: The Making of the Neoliberal Thought Collective, Cambridge, Harvard University Press, 2009 ; Daniel Stedman Jones, Masters of the universe : Hayek, Friedman, and the birth of neoliberal politics, Princeton, Princeton University Press, 2012.

17. Monica Prasad, The Politics of Free Markets: the Rise of Neoliberal Economic Policies in Britain, France, Germany, and the United States, Chicago, University of Chicago Press, 2006 ; Marion FourcadeGourinchas et Sarah Babb, "he Rebirth of the Liberal Creed: Paths to Neoliberalism in Four Countries", American Journal of Sociology, vol. 108, no 3, 2002, p. 533-579 ; Vivien A. Schmidt, "The Politics of Economic Adjustment in France and Britain: When Does Discourse Matter?", Journal of European Public Policy, vol. 8, nº 2, 2001, p. 247-264.

18. Sébastien Caré, «La dérive des continents néolibéraux : essai de typologie dynamique», Revue de philosophie économique, vol. 17, $\mathrm{n}^{\circ} 1,2016$, p. 21-55; Serge Audier, Néo-libéralisme(s) : une archéologie intellectuelle, Paris, Grasset, 2012.

19. Pierre Dardot et Christian Laval, La nouvelle raison du monde : essai sur la société néolibérale, Paris, la Découverte, 2009.

20. Du nom de la Société du Mont Pèlerin fondée par 
Friedrich Hayek en 1947.

21. Pour la présentation d'une approche compréhensive similaire à la nôtre pour étudier le néo-libéralisme, voir Gwendal Châton et Sebastien Caré, « Néolibéralisme (s) et démocratie (s) », op. cit.

22. Marion Fourcade-Gourinchas et Sarah Babb; John L. Campbell et Ove K. Pedersen, The National Origins of Policy Ideas: Knowledge Regimes in the United States, France, Germany, and Denmark, Princeton University Press, 2014..

23. À l'exception de François Denord dont les travaux portent majoritairement sur la période d'entre deuxguerres et d'immédiat après-guerre, François Denord, Le néo-libéralisme à la française: histoire d'une idéologie politique, Marseille, Agone, 2016.

24. Mark Blyth, Great Transformations: Economic Ideas and Institutional Change in the Twentieth Century, Cambridge, Cambridge University Press, 2002.

25. Raymond Boudon, Pourquoi les intellectuels n'aiment pas le libéralisme, Paris, Odile Jacob, 2004, p. 153.

26. Frédéric Sawicki et Johanna Siméant, «Décloisonner la sociologie de l'engagement militant. Note critique sur quelques tendances récentes des travaux français », Sociologie du travail, vol. 51, n¹, 2009, p. $97-125$.

\section{$\mathrm{R} \cdot \dot{E} \cdot \mathrm{S} \cdot \mathrm{U} \cdot \mathrm{M} \cdot \mathrm{E}$}

Ce numéro vise à interroger les modalités concrètes de la diffusion de l'idéologie néo-libérale dans le contexte institutionnel et politique français. Il accrode une attention particulère aux formes de mobilisation choisies pour militer pour ces idées. Qui sont les médiateurs du néo-libéralisme en France? Quels sont leurs répertoires d'actions et les formes de mobilisations privilégiées? Quels sont leurs liens avec les acteurs transnationaux de diffusion du néo-libéralisme et comment inscrivent-ils leur idéologie dans leur contexte national ? Le numéro montre la difficile diffusion de ces idées dans un contexte où le discours anticapitaliste est important. C'est à partir de mobilisations silencieuses et en adaptant leur discours au débat public que les militants néo-libéraux privilégient une approche incrémentale. Dans ce processus, on observe non seulement un transfert d'idées d'organisations internationales, mais également un transfert des formes de mobilisations militantes. Les contributions rendent compte de plusierus d'entre elles : la mise en avant d'un instrument de politique publique au niveau local (le chèque culture), le militantisme par l'expertise des think tanks, le cas des mobilisations des jeunes libertariens français sur le web, ainsi qu'un entretien avec Gaspard Koenig, figure contemporaine de la promotion de ces idées en France. 
